AJMS

28,1

\title{
Some remarks on invariant lightlike submanifolds of indefinite Sasakian manifold
}

100

Received 24 October 2020 Revised 27 January 2021 Accepted 1 March 2021

\author{
Samuel Ssekajja \\ School of Mathematics, University of the Witwatersrand, \\ Johannesburg, South Africa
}

\begin{abstract}
Purpose - The author considers an invariant lightlike submanifold $M$, whose transversal bundle $\operatorname{tr}(T M)$ is flat, in an indefinite Sasakian manifold $\bar{M}(c)$ of constant $\bar{\phi}$-sectional curvature $c$. Under some geometric conditions, the author demonstrates that $c=1$, that is, $\bar{M}$ is a space of constant curvature 1 . Moreover, $M$ and any leaf $M^{\prime}$ of its screen distribution $S(T M)$ are, also, spaces of constant curvature 1 .

Design/methodology/approach - The author has employed the techniques developed by K. L. Duggal and A. Bejancu of reference number 7 .

Findings - The author has discovered that any totally umbilic invariant ligtlike submanifold, whose transversal bundle is flat, in an indefinite Sasakian space form is, in fact, a space of constant curvature 1 (see Theorem 4.4).

Originality/value - To the best of the author's findings, at the time of submission of this paper, the results
\end{abstract} reported are new and interesting as far as lightlike geometry is concerned.

Keywords Lightlike submanifolds, Totally umbilic submanifolds, Invariant submanifolds,

Sasakian manifolds

Paper type Research paper

\section{Introduction}

Unlike non-degenerate submanifolds, lightlike submanifolds are quite complicated to study. One of the main reasons is that the tangent and normal bundles of a lightlike submanifold have, in general, a non-trivial intersection. It follows that one may not be able to use the wellknown structural equations for non-degenerate submanifolds on lightlike submanifolds. In trying to overcome such difficulties, K. L. Duggal and A. Bejancu published their work [1] on lightlike submanifolds of semi-Riemannian manifolds. Later, it was updated by K. L. Duggal and B. Sahin to reference [2]. In the above two books, the authors make use of a nondegenerate screen distribution on the submanifold, which gives rise to a four-factor breakdown of the ambient space. Unfortunately, the screen distribution is generally not unique and up to now there is no preferred technique of finding one. However, with some geometric conditions, one can secure a unique screen distribution, and some classes of lightlike submanifolds have been discussed, in the above books, with canonical screens, like the Monge lightlike hypersurfaces and many more. The foundations set in the books

JEL Classification — Primary 53C25; Secondary 53C40, 53C50

(C) Samuel Ssekajja. Published in Arab Journal of Mathematical Sciences. Published by Emerald Publishing Limited. This article is published under the Creative Commons Attribution (CC BY 4.0) licence. Anyone may reproduce, distribute, translate and create derivative works of this article (for both commercial and non-commercial purposes), subject to full attribution to the original publication and authors. The full terms of this licence may be seen at http://creativecommons.org/licences/by/4.0/ legalcode

The author wishes to thank the University of Witwatersrand for its generous financial support through a start-up research funding. The author also wishes to thank the anonymous referees for their comments and suggestions that greatly improved this paper. 
above motivated many other scholars to investigate the geometry of lightlike submanifolds. They include, amongst others, [3-16].

Theory of invariant non-degenerate submanifolds of almost-contact manifolds has extensively been studied and many interesting results are currently known about them. Some of the notable results on the topic can be found in references [17-19] and many more references cited therein. On the other hand, the invariant lightlike submanifolds have not yet been given the necessary attention. In fact, all the work presently known on this topic are limited to the scope set by K. L. Duggal and B. Sahin in the paper [20, pp. 4-6] as well as in the book [2, Chapter 7, p. 318]. Since invariant lightlike submanifolds are a part of many other general classes of lightlike submanifolds, such as the contact SCR (see [20, p. 11]), generalised CR [2, p. 334], amongst others, it would be important to understand their geometries well before any attempt is made to generalise them. The present paper is dedicated to the study of invariant lightlike submanifolds of indefinite Sasakian manifolds, whose transversal bundle is flat. The rest of the paper is arranged as follows: in Section 2, we quote some basics notions on almost-contact manifolds as well as lightlike geometry required in the rest of the paper. In Section 3, we focus on invariant submanifolds and some basic results. In Section 4, we discuss invariant submanifolds whose transversal bundles are flat in indefinite Sasakian space form.

\section{Preliminaries}

A $(2 \bar{n}+1)$-dimensional semi-Riemannian manifold $\bar{M}=(\bar{M}, \bar{g}, \bar{\phi}, \zeta, \eta)$ is said to be an indefinite Sasakian manifold [21] if it admits an almost-contact structure $(\bar{\phi}, \zeta, \eta)$, that is $\bar{\phi}$ is a tensor of type $(1,1)$ of rank $2 \bar{n}, \zeta$ is a unit spacelike vector field and $\eta$ is a 1 -form satisfying

$$
\begin{gathered}
\bar{\varphi}^{2}=-I+\eta \otimes \zeta, \quad \eta(\zeta)=1, \quad \eta(X)=\bar{g}(X, \zeta), \quad \bar{\phi} \zeta=0, \quad \eta^{\circ} \bar{\phi}=0, \\
\bar{g}(\bar{\varphi} X, \bar{\phi} Y)=\bar{g}(X, Y)-\eta(X) \eta(Y), \quad\left(\bar{\nabla}_{X} \bar{\phi}\right) Y=\bar{g}(X, Y) \zeta-\eta(Y) X, \\
\bar{\nabla}_{X} \zeta=-\bar{\phi} X, \quad \bar{R}(X, Y) \zeta=\eta(Y) X-\eta(X) Y,
\end{gathered}
$$

for all $X$ and $Y$ tangent to $\bar{M}$. Here, $\bar{\nabla}$ is the Levi-Civita connection for a semi-Riemannian metric $\bar{g}$. Furthermore, $\bar{R}$ is the curvature tensor of $\bar{M}$. Next, a plane section $\pi$ in $T_{x} \bar{M}$ of a Sasakian manifold $\bar{M}$ is called a $\bar{\phi}$-section if it is spanned by a unit vector $X$ orthogonal to $\zeta$ and $\bar{\phi} X$, where $X$ is a non-null vector field on $\bar{M}$. The sectional curvature $\bar{\kappa}(X, \bar{\phi} X)$ of a $\bar{\phi}$-section is called a $\bar{\phi}$-sectional curvature. When $c$ does not depend on the $\bar{\phi}$-section at each point, then $c$ constant in $\bar{M}$ and $\bar{M}$ is called a Sasakian space form, denoted by $\bar{M}(c)$. Moreover, the curvature tensor $\bar{R}$ of $\bar{M}$ satisfies (see [2, Theorem 7.1.3, p. 307])

$$
\begin{aligned}
4 \bar{R}(X, Y, Z, W)= & (c+3)\{\bar{g}(Y, Z) \bar{g}(X, W)-\bar{g}(X, Z) \bar{g}(Y, W)\} \\
& +(c-1)\{\eta(X) \eta(Z) \bar{g}(Y, W)-\eta(Y) \eta(Z) \bar{g}(X, W) \\
& +\bar{g}(X, Z) \eta(Y) \eta(W)-\bar{g}(Y, Z) \eta(X) \eta(W) \\
& +\bar{g}(\bar{\phi} Y, Z) \bar{g}(\bar{\phi} X, W)-\bar{g}(\bar{\phi} X, Z) \bar{g}(\bar{\phi} Y, W) \\
& -2 \bar{g}(\bar{\phi} X, Y) \bar{g}(\bar{\phi} Z, W)\}, \forall X, Y, Z, W \in T(T \bar{M})
\end{aligned}
$$

Let $(\bar{M}, \bar{g})$ be a real $(m+n)$-dimensional semi-Riemannian manifold, where $m>1$ and $n \geq 1$, with $\bar{g}$ a semi-Riemannian metric of index $q$, such that $1 \leq q \leq m+n-1$. It follows that $\bar{M}$ is never a Riemannian manifold. Let $M$ be an $m$-dimensional submanifold of $\bar{M}$. For each $p \in M$,
Invariant lightlike submanifolds 
AJMS

28,1

102

we consider $T_{p} M^{\perp}=\left\{U_{p} \in T_{p} \bar{M}: \bar{g}_{p}\left(U_{p}, X_{p}\right)=0, \forall X_{p} \in T_{p} M\right\}$. If $M$ is a lightlike submanifold, then there exists a smooth distribution $\operatorname{Rad} T_{p} M$, called the radical distribution, such that $\operatorname{Rad} T_{p} M=T_{p} M \cap T_{p} M^{\perp} \neq\{0\}$, for all $p \in M$. Denote by $r$ the rank of $\operatorname{Rad} T M$. If $r>0$, then $M$ is called an $r$-lightlike submanifold [2, p. 191]. There are four possible classes of lightlike submanifolds, according to

(1) $r$-lightlike submanifold, $0<r<\min \{m, n\}$,

(2) co-isotropic submanifold, $1<r=n<m$,

(3) isotropic submanifold, $1<r=m<n$,

(4) totally lightlike submanifold, $1<r=n=m$.

Next, we consider a complementary distribution to $\operatorname{Rad} T M$ in $T M$, called the screen distribution and denoted by $S(T M)$. Such a screen is always secured due to the fact that $M$ is paracompact. Moreover, $S(T M)$ is orthogonal to $\operatorname{Rad} T M$ and non-degenerate with respect to $\bar{g}$. Thus, we have the decomposition $T M=S(T M) \perp \operatorname{Rad} T M$. Obviously, $S(T M)$ is not unique; however, it is canonically isomorphic to the factor bundle $T M / \operatorname{Rad} T M$ [22]. Let us consider the vector bundle $T M^{\perp}=\underset{p \in M}{\cup} T_{p} M^{\perp}$. In a lightlike case, $T M^{\perp}$ is not complementary to $T M$ in $\left.T \bar{M}\right|_{M}$ due to the fact that $\operatorname{Rad} T M=T M \cap T M^{\perp}$ is a distribution on $M$ of rank $r>1$. Next, let us consider a non-degenerate complementary vector bundle $S\left(T M^{\perp}\right)$ to $\operatorname{Rad} T M$ in $T M^{\perp}$. Then, $T M^{\perp}=S\left(T M^{\perp}\right) \perp \operatorname{Rad} T M$. We call $S\left(T M^{\perp}\right)$ the screen transversal bundle of $M$. Furthermore, using the fact that $S(T M)$ is non-degenerate, we have the decomposition $T \bar{M}_{\mid M}=S(T M) \perp S(T M)^{\perp}$, where $S(T M)^{\perp}$ is the complementary orthogonal vector bundle to $S(T M)$ in $T \bar{M}_{\mid M}$. Note that $S\left(T M^{\perp}\right)$ is a vector subbundle of $S(T M)^{\perp}$, and since both are non-degenerate, we have the orthogonal decomposition $S(T M)^{\perp}=S\left(T M^{\perp}\right) \perp S\left(T M^{\perp}\right)^{\perp}$. The theory of lightlike submanifolds largely depends on the vector bundles $S(T M)$ and $S\left(T M^{\perp}\right)$, a lightlike submanifold is often denoted as $\left(M, g, S(T M), S\left(T M^{\perp}\right)\right)$. The following characterisation result of lightlike submanifolds is well known:

Theorem 2.1. (Duggal-Sahin [2]). Let $\left(M, g, S(T M), S\left(T M^{\perp}\right)\right.$ ) be an $r$-lightlike submanifold of semi-Riemannian manifold $(\bar{M}, \bar{g})$. Suppose $\mathcal{U}$ is a coordinate neighbourhood of $M$. There exists a complementary vector bundle $l \operatorname{tr}(T M)$, called the lightlike transversal bundle of $\operatorname{Rad} T M$ in $S\left(T M^{\perp}\right)^{\perp}$ and a basis of $\Gamma\left(l \operatorname{tr}(T M)_{\mathcal{U}}\right)$ consists of smooth sections $\left\{N_{1}, \ldots, N_{r}\right\}$ of $S\left(T M^{\perp}\right)_{\mid \mathcal{U}}^{\perp}$ such that $\bar{g}\left(\xi_{i}, N_{j}\right)=\delta_{i j}, \bar{g}\left(N_{i}, N_{j}\right)=0$, $i, j=1, \ldots, r$, where $\left\{\xi_{1}, \ldots, \xi_{r}\right\}$ is a basis of $\Gamma(\operatorname{Rad} T M)$.

The above theorem shows that there exists a complementary (but not orthogonal) vector bundle $\operatorname{tr}(T M)$ to $T M$ in $T \bar{M}_{\mid M}$, called the transversal bundle, such that $\operatorname{tr}(T M)=$ $l \operatorname{tr}(T M) \perp S\left(T M^{\perp}\right)$ and $T \bar{M}_{\mid M}=T M \oplus \operatorname{tr}(M)$.

From now on, we denote by $M$ an $m$-dimensional lightlike submanifold instead of $\left(M, g, S(T M), S\left(T M^{\perp}\right)\right)$ and $(m+n)$-dimensional semi-Riemannian manifold by $\bar{M}$. Let us denote by $F(M)$ the algebra of smooth functions on $M$ and $\Gamma(E)$ the $F(M)$ module of smooth sections of a vector bundle $E$ (the same notation for any other vector bundle) over $M$. Then, we have

$$
\begin{gathered}
\bar{\nabla}_{X} Y=\nabla_{X} Y+h(X, Y), \forall X, Y \in \Gamma(T M), \\
\bar{\nabla}_{X} U=-A_{U} X+\nabla_{X}^{t} U, \forall X \in \Gamma(T M), U \in \Gamma(\operatorname{tr}(T M)),
\end{gathered}
$$


where $\left\{\nabla_{X} Y, A_{U} X\right\}$ and $\left\{h(X, Y), \nabla_{X}^{t} U\right\}$ belong to $\Gamma(T M)$ and $\Gamma(\operatorname{tr}(T M))$, respectively. Further, $\nabla$ and $\nabla^{t}$ are linear connections on $M$ and $\operatorname{tr}(T M)$, respectively. The second fundamental form $h$ is a symmetric $F(M)$-bilinear form on $\Gamma(T M)$ with values in $\Gamma(\operatorname{tr}(T M))$ and the shape operator $A_{V}$ is a linear endomorphism of $\Gamma(T M)$. Moreover, (2.5) and (2.6) lead to (see [2, pp. 196-198]).

$$
\begin{gathered}
\bar{\nabla}_{X} Y=\nabla_{X} Y+h^{l}(X, Y)+h^{s}(X, Y), \\
\bar{\nabla}_{X} N=-A_{N} X+\nabla_{X}^{l} N+D^{s}(X, N), \\
\bar{\nabla}_{X} W=-A_{W} X+\nabla_{X}^{s} W+D^{l}(X, W),
\end{gathered}
$$

for all $X, Y \in \Gamma(T M), N \in \Gamma(l \operatorname{tr}(T M))$ and $W \in \Gamma\left(S\left(T M^{\perp}\right)\right)$. Here, $A_{N}$ and $A_{W}$ are called the shape operators of $M$. We call $h^{l}$ and $h^{s}$ the lightlike second fundamental form and the screen second fundamental form, respectively. Furthermore, $\nabla^{l}$ and $\nabla^{s}$ are, respectively, linear connections on $l \operatorname{tr}(T M)$ and $S\left(T M^{\perp}\right)$, called the lightlike connection and the screen transversal connection. Note that $D^{l}$ and $D^{s}$ are Otsuki connections on $l \operatorname{tr}(T M)$ and $S\left(T M^{\perp}\right)$, respectively. Denote the projection of $T M$ on $S(T M)$ by $P$. Then, we have

$$
\nabla_{X} P Y=\nabla_{X}^{*} P Y+h^{*}(X, P Y), \nabla_{X} \xi=-A_{\xi}^{*} X+\nabla_{X}^{* t} \xi,
$$

for all $X, Y \in \Gamma(T M)$ and $\xi \in \Gamma(\operatorname{Rad} T M)$. Here, $\nabla^{*}$ and $A_{\xi}^{*}$ are, respectively, the linear connection and shape operator of $S(T M)$. Furthermore, $h^{*}$ and $\nabla^{* t}$ stand for the second fundamental form and a linear connection on $\operatorname{Rad} T M$, respectively. Furthermore, by using (2.5), (2.7)-(2.10), we obtain

$$
\begin{gathered}
\bar{g}\left(h^{s}(X, Y), W\right)+\bar{g}\left(Y, D^{l}(X, W)\right)=g\left(A_{W} X, Y\right), \\
\bar{g}\left(h^{l}(X, Y), \xi\right)+\bar{g}\left(Y, h^{l}(X, \xi)\right)+g\left(Y, \nabla_{X} \xi\right)=0, \\
\bar{g}\left(h^{*}(X, P Y), N\right)=g\left(A_{N} X, P Y\right), \quad \bar{g}\left(h^{l}(X, \xi), \xi\right)=0, \quad A_{\xi}^{*} \xi=0, \\
\bar{g}\left(D^{s}(X, N), W\right)=\bar{g}\left(N, A_{W}, X\right), \quad \bar{g}\left(\bar{\nabla}_{X} P Y, N\right)=g\left(P Y, A_{N} X\right),
\end{gathered}
$$

where $X, Y \in \Gamma(T M), \xi \in \Gamma(\operatorname{Rad} T M)$ and $W \in \Gamma\left(S\left(T M^{\perp}\right)\right)$. In general, the induced connection $\nabla$ on $M$ is not a metric connection. Since $\bar{\nabla}$ is a metric connection, by using (2.7), we get $\left(\nabla_{X} g\right)(Y, Z)=\bar{g}\left(h^{l}(X, Y), Z\right)+\bar{g}\left(h^{l}(X, Z), Y\right)$, for all $X, Y, Z \in \Gamma(T M)$. However, it is important to note that $\nabla^{*}$ is a metric connection on $S(T M)$. Denoted by $R, R^{l}$ and $R^{s}$, the curvature tensors of $M, l \operatorname{tr}(T M)$ and $S\left(T M^{\perp}\right)$, respectively. Then we have (see $[1$, p. 171] for more details)

$$
\begin{aligned}
\bar{R}(X, Y) Z= & R(X, Y) Z+A_{h^{l}(X, Z)} Y-A_{h^{l}(Y, Z)} X+A_{h^{s}(X, Z)} Y \\
& -A_{h^{s}(Y, Z)} X+\left(\widetilde{\nabla}_{X} h^{l}\right)(Y, Z)-\left(\widetilde{\nabla}_{Y} h^{l}\right)(X, Z)+D^{l}\left(X, h^{s}(Y, Z)\right) \\
& -D^{l}\left(Y, h^{s}(X, Z)\right)+\left(\widetilde{\nabla}_{X} h^{s}\right)(Y, Z)-\left(\widetilde{\nabla}_{Y} h^{s}\right)(X, Z) \\
& +D^{s}\left(X, h^{l}(Y, Z)\right)-D^{s}\left(Y, h^{l}(X, Z)\right),
\end{aligned}
$$

Invariant lightlike submanifolds 
AJMS

28,1

\section{4}

$$
\begin{aligned}
\bar{R}(X, Y) N= & R^{l}(X, Y) N+h^{l}\left(Y, A_{N} X\right)-h^{l}\left(X, A_{N} Y\right)+D^{l}\left(X, D^{s}(Y, N)\right) \\
& -D^{l}\left(Y, D^{s}(X, N)\right)+\left(\nabla_{Y} A\right)(N, X)-\left(\nabla_{X} A\right)(N, Y) \\
& +A_{D^{s}(X, N)} Y-A_{D^{s}(Y, N)} X+\left(\nabla_{X} D^{s}\right)(Y, N)-\left(\nabla_{Y} D^{s}\right)(X, N) \\
& +h^{s}\left(Y, A_{N} X\right)-h^{s}\left(X, A_{N} Y\right)
\end{aligned}
$$

where $\widetilde{\nabla} h^{l}, \widetilde{\nabla} h^{s}, \nabla D^{l}, \nabla D^{s},\left(\nabla_{X} A\right)(Y, N)$ and $\left(\nabla_{X} A\right)(Y, W)$ are given by

$$
\begin{gathered}
\left(\widetilde{\nabla}_{X} h^{l}\right)(Y, Z)=\nabla_{X}^{l} h^{l}(Y, Z)-h^{l}\left(\nabla_{X} Y, Z\right)-h^{l}\left(Y, \nabla_{X} Z\right), \\
\left(\widetilde{\nabla}_{X} h^{s}\right)(Y, Z)=\nabla_{X}^{s} h^{s}(Y, Z)-h^{s}\left(\nabla_{X} Y, Z\right)-h^{s}\left(Y, \nabla_{X} Z\right), \\
\left(\nabla_{X} D^{l}\right)(Y, W)=\nabla_{X}^{l} D^{l}(Y, W)-D^{l}\left(\nabla_{X} Y, W\right)-D^{l}\left(Y, \nabla_{X}^{s} W\right), \\
\left(\nabla_{X} D^{s}\right)(Y, N)=\nabla_{X}^{s} D^{s}(Y, N)-D^{s}\left(\nabla_{X} Y, N\right)-D^{s}\left(Y, \nabla_{X}^{l} N\right), \\
\left(\nabla_{X} A\right)(N, Y)=\nabla_{X} A(N, Y)-A\left(\nabla_{X}^{l} N, Y\right)-A\left(N, \nabla_{X} Y\right), \\
\left(\nabla_{X} A\right)(W, Y)=\nabla_{X} A(W, Y)-A\left(\nabla_{X}^{l} W, Y\right)-A\left(W, \nabla_{X} Y\right),
\end{gathered}
$$

for all $X, Y, Z \in \Gamma(T M), N \in \Gamma(\operatorname{tr}(T M))$ and $W \in \Gamma\left(S\left(T M^{\perp}\right)\right)$. Furthermore, we say that the screen transversal bundle $S\left(T M^{\perp}\right)$ is flat if $\nabla^{s}$ is a flat linear connection. In this case, the corresponding curvature tensor $R^{s}$ vanishes. Similarly, the lightlike transversal bundle $l \operatorname{tr}(T M)$ is flat if $\nabla^{l}$ is a flat linear connection, which also implies that $R^{l}$ vanishes. Next, we end this section by defining the parallelism of the connections $D^{l}$ and $D^{s}$.

Definition 2.2. We say that the Otsuki connection $D^{l}$ (resp. $D^{s}$ ) is parallel if $\nabla D^{l}=0$ (resp. $\left.\nabla D^{s}=0\right)$.

It follows from relations (2.20), (2.21) and Definition 2.2 that $D^{l}$ and $D^{s}$ are parallel if and only if

$$
\begin{gathered}
\nabla_{X}^{l} D^{l}(Y, W)=D^{l}\left(\nabla_{X} Y, W\right)+D^{l}\left(Y, \nabla_{X}^{s} W\right), \\
\text { and } \nabla_{X}^{s} D^{s}(Y, N)=D^{s}\left(\nabla_{X} Y, N\right)+D^{s}\left(Y, \nabla_{X}^{l} N\right),
\end{gathered}
$$

for all $X, Y \in \Gamma(T M)$, respectively.

\section{Definitions and basic results}

Let $M$ be a lightlike submanifold of an indefinite almost-contact metric manifold $\bar{M}=(\bar{M}, \zeta, \eta, \bar{\phi}, \bar{g})$. If $\zeta$ is tangent to $M$, then $\zeta$ does not belong to the lightlike distribution $\operatorname{Rad} T M$. Thus, by $\zeta$ tangent, we shall mean $\zeta \in \Gamma(S(T M))$ [8]. With the above note in mind, we have the following definition: 
Definition 3.1. (Duggal-Sahin [2]). Let $M$ be a lightlike submanifold of an indefinite Sasakian manifold $\bar{M}$, tangent to $\zeta$, that is, $\zeta \in \Gamma(S(T M))$. We call $M$ an invariant lightlike submanifold if both $S(T M)$ and $\operatorname{Rad} T M$ are invariant with respect to $\bar{\phi}$. That is, $\bar{\phi} S(T M)=S(T M)$ and $\bar{\phi} \operatorname{Rad} T M=\operatorname{Rad} T M$.

It is easy to see, from Definition 3.1 above, that $l \operatorname{tr}(T M)$ and $S\left(T M^{\perp}\right)$ are also invariant with respect to $\bar{\phi}$. That is, $\bar{\phi} \operatorname{tr}(T M)=l \operatorname{tr}(T M)$ and $\bar{\phi} S\left(T M^{\perp}\right)=S\left(T M^{\perp}\right)$. Also the following, about an invariant lightlike submanifold, holds:

Proposition 3.2. There exist no any isotropic or totally lightlike invariant submanifold $M$ of an indefinite Sasakian manifold $\bar{M}$.

According to Proposition 3.2, by an invariant lightlike submanifold $M$ of an indefinite Sasakian manifold $\bar{M}$, we shall always mean $M$ to be an $r$-lightlike or a co-isotropic in $\bar{M}$.

Lemma 3.3. Let $M$ be an invariant lightlike submanifold of an indefinite Sasakian manifold. Then, the following holds:

$$
\begin{gathered}
\nabla_{X} \zeta=-\bar{\phi} X, \quad h^{l}(X, \zeta)=h^{s}(X, \zeta)=0, \\
h(X, \bar{\phi} Y)=\bar{\phi} h(X, Y), \quad h(\bar{\phi} X, \bar{\phi} Y)=-h(X, Y), \\
\left(\nabla_{X} \bar{\phi}\right) Y=g(X, Y) \zeta-\eta(Y) X, \quad R(X, Y) \zeta=\eta(Y) X-\eta(X) Y,
\end{gathered}
$$

for all $X, Y \in \Gamma(T M)$.

Proof: The relations in (3.1), (3.2) and the first in (3.3) follow easily from (2.7), (2.2) and (2.3). Turning to the second relation in (3.3). Setting $Z=\zeta$ in (2.15) and then considering (3.1) in the resulting relation, we get

$$
\begin{aligned}
\bar{R}(X, Y) \zeta= & R(X, Y) \zeta+\left(\nabla_{X} h^{l}\right)(Y, \zeta) \\
& -\left(\nabla_{Y} h^{l}\right)(X, \zeta)+\left(\nabla_{X} h^{s}\right)(Y, \zeta)-\left(\nabla_{Y} h^{s}\right)(X, \zeta)
\end{aligned}
$$

for any $X, Y \in \Gamma(T M)$. Now, using (2.18) and (3.1), we derive $\left(\nabla_{X} h^{l}\right)(Y, \zeta)=-h^{l}\left(Y, \nabla_{X} \zeta\right)$ $=h^{l}(Y, \bar{\phi} X)$. It then follows

$$
\left(\nabla_{X} h^{l}\right)(Y, \zeta)-\left(\nabla_{Y} h^{l}\right)(X, \zeta)=0
$$

in which we have used (3.2) and the symmetry of $h^{l}$. In a similar way, but this time using (2.19) and (3.1), we have $\left(\nabla_{X} h^{s}\right)(Y, \zeta)=h^{s}(Y, \bar{\phi} X)$, from which

$$
\left(\nabla_{X} h^{s}\right)(Y, \zeta)-\left(\nabla_{Y} h^{s}\right)(X, \zeta)=0 .
$$

Then, putting (3.5) and (3.6) in (3.4), we obtain $\bar{R}(X, Y) \zeta=R(X, Y) \zeta$. It then follows from (2.3) that $R(X, Y) \zeta=\eta(Y) X-\eta(X) Y$.

Considering Lemma 3.3, we have the following.

Theorem 3.4. The sectional curvature of any non-degenerate plane spanned by $\zeta$ and a non-null vector field on $M$ orthogonal to $\zeta$ is 1 .

Lemma 3.5. On any invariant lightlike submanifold $M$ of an indefinite Sasakian manifold $\bar{M}$, we have the following:

(1) $A_{\bar{\phi} N} X=\bar{\phi} A_{N} X-\bar{g}(X, N) \zeta, \nabla_{X}^{l} \bar{\phi} N=\bar{\phi} \nabla_{X}^{l} N, D^{s}(X, \bar{\phi} N)=\bar{\phi} D^{s}(X, N)$; 
AJMS

28,1

(2) $A_{\bar{\phi} W} X=\bar{\phi} A_{W} X, \nabla_{X}^{s} \bar{\phi} W=\bar{\phi} \nabla_{X}^{s} W, D^{l}(X, \bar{\phi} W)=\bar{\phi} D^{l}(X, W)$, for any $X \in \Gamma(T M)$.

Proof: Taking $Y=N$ in the second relation of (2.2), we have $\left(\bar{\nabla}_{X} \bar{\phi}\right) N=\bar{g}(X, N) \zeta$, for any $X \in \Gamma(T M)$. Then, applying (2.8) to this relation leads to

$$
-A_{\bar{\phi} N} X+\nabla_{X}^{l} \bar{\phi} N+D^{s}(X, \bar{\phi} N)+\bar{\phi} A_{N} X-\bar{\phi} \nabla_{X}^{l} N-\bar{\phi} D^{s}(X, N)=\bar{g}(X, N) \zeta .
$$

The relations in (1), then, follow from (3.7) by comparing tangential and transversal parts. On the other hand, using (2.3) and (2.9), we derive

$$
-A_{\bar{\phi} W} X+\nabla_{X}^{s} \bar{\phi} W+D^{l}(X, \bar{\phi} W)+\bar{\phi} A_{W} X-\bar{\phi} \nabla_{X}^{s} W-\bar{\phi} D^{l}(X, W)=0,
$$

for all $X \in \Gamma(T M)$. Finally, the relations in (2) follow from (3.8) by comparing tangential and transversal parts.

From the first relation in (1) of Lemma 3.5, the following holds:

Proposition 3.6. There exists no any invariant lightlike submanifold of an indefinite Sasakian manifold such that $A_{N}$ vanishes on $\operatorname{Rad} T M$.

It is well known [9, Eq. 4.20, p. 62] that when $S(T M)$ is totally umbilic, then

$$
P A_{N} X=\lambda P X \text { and } h^{*}(\xi, P X)=0,
$$

for any $X \in \Gamma(T M)$, where $\lambda$ is smooth function on each coordinate neighbourhood $\mathcal{U} \subset M$. Thus, in case $M$ is invariant submanifold, with a totally umbilic screen, the first relation in (1) of Lemma 3.5 and the first relation of (3.9) gives

$$
P \bar{\phi} A_{N} \xi=\bar{g}(\xi, N) \zeta
$$

Taking the inner product of (3.10) with $\zeta$ leads to $\bar{g}(\xi, N)=g\left(P \bar{\phi} A_{N} \xi, \zeta\right)=$ $g\left(\bar{\phi} A_{N} \xi, \zeta\right)=0$. This is clearly a contradiction.

On the other hand, when $S(T M)$ is parallel, with respect to $\nabla$, it is known [2, p. 89] that $h^{*}(X, P Y)=0$, for all $X, Y \in \Gamma(T M)$. From this relation and the first one in (2.13), we see that

$$
g\left(A_{N} \xi, P X\right)=0,
$$

for each $X \in \Gamma(T M)$ and $N \in \Gamma(l \operatorname{tr}(T M))$. Then, the first relation in (1) of Lemma 3.5, leads to

$$
g\left(A_{\bar{\phi} N} \xi, P Y\right)=-g\left(A_{N} \xi, \bar{\phi} P Y\right)-\bar{g}(\xi, N) g(\zeta, P Y) .
$$

Thus, from (3.11) and (3.12), we have $\bar{g}(\xi, N) g(\zeta, P Y)=0$, for any $Y \in \Gamma(T M)$. As $\bar{g}(\xi, N) \neq 0$, it follows that $g(\zeta, P Y)=0$. Now, replacing $P Y$ with $\zeta$ (this is possible since $\zeta$ belongs to $S(T M)$ by Definition 3.1) in the last relation, we get $g(\zeta, \zeta)=0$, which is a contradiction to $g(\zeta, \zeta)=\eta(\zeta)=1$ (see the second relation in (2.1). With the above discussion, we have the following result:

Theorem 3.7. There exists no any invariant lightlike submanifold of an indefinite Sasakian manifold with a totally umbilic or parallel screen distribution.

Lemma 3.8. Let $M$ be an invariant lightlike submanifold of an indefinite Sasakian manifold $\bar{M}$. Then, the following holds:

(1) $D^{l}(\zeta, W)=0$.

(2) $D^{s}(\zeta, N)=0$ if and only if $A_{W} \zeta=0$. 
Proof: From (2.11) and (3.1), we have $\bar{g}\left(Y, D^{l}(\zeta, W)\right)=g\left(A_{W} \zeta, Y\right)$, for all $Y \in \Gamma(T M)$. Taking $Y=\xi$ in this relation, we get $\bar{g}\left(\xi, D^{l}(\zeta, W)\right)=0$. It follows from the last relation that $D^{l}(\zeta, W)=0$. On the other hand, from $(2.11)$, we have $\bar{g}\left(h^{s}(X, P Y), W\right)=g\left(A_{W} X, P Y\right)$, for any $X, Y \in \Gamma(T M)$. Taking $X=\zeta$ in this relation and then applying in (2.15), we get $g\left(A_{W} \zeta, P Y\right)=0$. It follows from this relation that $A_{W} \zeta$ is $\Gamma(\operatorname{Rad} T M)$ valued. Hence, using this information in the first relation of (2.14), we conclude that $D^{\varsigma}(\zeta, N)=0$ if and only if $A_{W} \zeta=0$.

Proposition 3.9. Let $M$ be an invariant lightlike submanifold of an indefinite Sasakian manifold $\bar{M}$. Then,

(1) $D^{l}$ is parallel if and only if $D^{l}=0$. Moreover, $A_{W}$ is a symmetric operator.

(2) $D^{s}$ is parallel and $A_{W} \zeta=0$ if and only if $D^{s}=0$.

Proof: Using (2.22) and Lemma 3.8, we have $D^{l}\left(\nabla_{X} \zeta, W\right)=0$, for any $X \in \Gamma(T M)$. Now, applying the first relation of (3.1) to this, we get $D^{l}(\bar{\phi} X, W)=0$. Replacing $X$ with $\bar{\phi} X$, we get $-D^{l}(X, W)+\eta(X) D(\zeta, W)=-D^{l}(X, W)=0$. Hence, $D^{l}=0$. Now from (2.11), we have $\bar{g}\left(h^{s}(X, Y), W\right)=g\left(A_{W} X, Y\right)$, for any $X, Y \in \Gamma(T M)$. Since $h^{s}$ is symmetric, it follows that $A_{W}$ is symmetric on $T M$, which proves (1). The proof of (2) follows similar steps, while considering (2.23), which completes the proof.

\section{Main results}

In this section, we characterise an invariant lightlike submanifold $M$ of an indefinite Sasakian manifold $\bar{M}$, whose transversal bundle is flat. In line with the above, we start with a few characterisation results.

Proposition 4.1. Let $M$ be an invariant lightlike submanifold of an indefinite Sasakian space form $\bar{M}(c)$, with a flat screen transversal bundle $S\left(T M^{\perp}\right)$. Then,

$$
\begin{aligned}
& (c-1) \bar{g}(W, W) g(\bar{\varphi} X, \bar{\varphi} Y)=-2\left\{\bar{g}\left(h^{s}\left(Y, A_{W} X\right), W\right)\right. \\
& \quad+\bar{g}\left(h^{s}\left(X, A_{\bar{\varphi} W} \bar{\varphi} Y\right), W\right)-\bar{g}\left(D^{s}\left(X, D^{l}(\bar{\varphi} Y, \bar{\varphi} W)\right), W\right) \\
& \left.\quad+\bar{g}\left(D^{s}\left(\bar{\varphi} Y, D^{l}(X, \bar{\varphi} W)\right), W\right)\right\},
\end{aligned}
$$

for all $X, Y \in \Gamma(T M)$ and $W \in \Gamma\left(S\left(T M^{\perp}\right)\right)$.

Proof: Replacing $Y$ with $\bar{\phi} Y, Z$ with $W$ and $W$ with $\bar{\phi} W$ in (2), we get

$$
\bar{R}(X, \bar{\phi} Y, W, \bar{\phi} W)=-\frac{c-1}{2} \bar{g}(W, W) g(\bar{\phi} X, \bar{\phi} Y),
$$

for all $X, Y \in \Gamma(T M)$. On the other hand, since $R^{s}=0$, (2.17) leads to

$$
\begin{aligned}
\bar{R}(X, \bar{\phi} Y, W, \bar{\phi} W)= & \bar{g}\left(h^{s}\left(\bar{\phi} Y, A_{W} X\right)-h^{s}\left(X, A_{W} \bar{\phi} Y\right), \bar{\phi} W\right)+\bar{g}\left(D^{s}\left(X, D^{l}(\bar{\phi} Y, W)\right)\right. \\
& \left.-D^{s}\left(\bar{\phi} Y, D^{l}(X, W)\right), \bar{\phi} W\right) .
\end{aligned}
$$

Then, applying the relations of Lemma 3.5 to (4.2), we get 
AJMS

28,1

$$
\begin{aligned}
\bar{R}(X, \bar{\phi} Y, W, \bar{\phi} W)= & \bar{g}\left(h^{s}\left(Y, A_{W} X\right), W\right)+\bar{g}\left(h^{s}\left(X, A_{\bar{\phi} W} \bar{\phi} Y\right), W\right) \\
& -\bar{g}\left(D^{s}\left(X, D^{l}(\bar{\phi} Y, \bar{\phi} W)\right), W\right)+\bar{g}\left(D^{s}\left(\bar{\phi} Y, D^{l}(X, \bar{\phi} W)\right), W\right),
\end{aligned}
$$

for any $X, Y \in \Gamma(T M)$ and $W \in \Gamma\left(S\left(T M^{\perp}\right)\right)$. Finally, our claim follows from (4.1) and (4.3), which completes the proof.

Proposition 4.2. Let $M$ be an invariant $r$-lightlike submanifold of an indefinite Sasakian space form $\bar{M}(c)$, with a flat screen transversal bundle $S\left(T M^{\perp}\right)$. If $D^{l}$ is parallel, then $\bar{M}(c)$ is a space of constant curvature $c=1$ if and only if $A_{W}$ has no components in $S(T M)$.

Proof: Suppose that $D^{l}$ is parallel. It follows from Proposition 3.9 that $A_{W}$ is a symmetric operator. Hence, applying Lemma 3.5 and (2.15), we derive,

$$
\bar{g}\left(h^{s}\left(X, A_{\bar{\phi} W} \bar{\phi} Y\right), W\right)=g\left(A_{W} X, A_{\bar{\phi} W} \bar{\phi} Y\right)=g\left(A_{W} X, A_{W} Y\right),
$$

for all $X, Y \in \Gamma(T M)$. Applying (4.4) to the relation of Proposition 4.1, we get,

$$
(c-1) \bar{g}(W, W) g(\bar{\phi} X, \bar{\phi} Y)=-4 g\left(A_{W} X, A_{W} Y\right) .
$$

Now, from (4.5), we see that when $c=1$, then $g\left(A_{W} X, A_{W} Y\right)=0$. This shows that $P A_{W} X=0$. On the other hand, when $P A_{W} X=0$, for each $X \in \Gamma(T M)$, then (4.5) gives $(c-1) \bar{g}(W, W) g(\bar{\phi} X, \bar{\phi} Y)=0$. Clearly, $c=1$ since $S(T M)$ and $S\left(T M^{\perp}\right)$ are nondegenerate subbundles, which completes the proof.

Proposition 4.3. Let $M$ be an invariant lightlike submanifold of an indefinite Sasakian manifold $\bar{M}$, such that $D^{l}$ is parallel. If the lightlike transversal bundle $l \operatorname{tr}(T M)$ is flat, then $c=1$ if and only if the operator $A_{N}$ is symmetric with respect to the lightlike second fundamental form $h^{l}$.

Proof: As $D^{l}$ is parallel, Proposition 3.9 suggests that $D^{l}=0$. Since $l \operatorname{tr}(T M)$ is flat, (2.16) leads to

$$
\bar{R}(X, Y, N, \xi)=\bar{g}\left(h^{l}\left(Y, A_{N} X\right)-h^{l}\left(X, A_{N} Y\right), \xi\right),
$$

for any $X, Y \in \Gamma(T M)$. Next, applying (4.6) to (2), we get

$$
\frac{(c-1)}{2} \bar{g}(X, \bar{\phi} Y) \bar{g}(\bar{\phi} N, \xi)=\bar{g}\left(h^{l}\left(Y, A_{N} X\right)-h^{l}\left(X, A_{N} Y\right), \xi\right) .
$$

If $c=1$, (4.7) leads to $\bar{g}\left(h^{l}\left(Y, A_{N} X\right)-h^{l}\left(X, A_{N} Y\right), \xi\right)=0$, from which we get $h^{l}\left(Y, A_{N} X\right)=h^{l}\left(X, A_{N} Y\right)$. Hence, $A_{N}$ is symmetric with respect to $h^{l}$. On the other hand, when $A_{N}$ is sympathetic with respect to $h^{l}$, (4.7) gives $(c-1) \bar{g}(X, \bar{\phi} Y) \bar{g}(\bar{\phi} N, \xi)=0$. Since $S(T M)$ is non-degenerate, we deduce that $c=1$, which completes the proof.

A lightlike submanifold $M$ of a semi-Riemannian manifold $\bar{M}$ is said to be totally umbilic [9, Definition 1, p. 58], in $\bar{M}$, if there is a smooth transversal vector field $H \in \Gamma(\operatorname{tr}(T M))$, called the transversal curvature vector field of $M$, such that $h(X, Y)=g(X, Y) H$, for all $X, Y \in \Gamma(T M)$. Moreover, $M$ is totally umbilic if and only if on each coordinate neighbourhood $\mathcal{U}$ there exist smooth vector fields $H^{l} \in \Gamma(\operatorname{lt}(T M))$ and $H^{s} \in \Gamma\left(S\left(T M^{\perp}\right)\right)$ such that $h^{l}(X, Y)=g(X, Y) H^{l}$ and $h^{s}(X, Y)=g(X, Y) H^{s}$. Furthermore, Theorem 4.1 of 
[9, p. 59] indicates that when $M$ is totally umbilic, then the Otsuki connection $D^{l}$, on $l \operatorname{tr}(T M)$, vanishes, that is, $D^{l}=0$. We say that $M$ is totally geodesic if $H$ vanishes, equivalently when both $H^{l}$ and $H^{s}$ vanish.

Theorem 4.4. Let $M$ be a totally umbilic invariant lightlike submanifold of an indefinite Sasakian space form $\bar{M}(c)$. If the lightlike transversal bundle $l \operatorname{tr}(T M)$ or the screen transversal bundle $S\left(T M^{\perp}\right)$ is flat, then $c=1$. Moreover,

(1) $M$ is a space of constant curvature 1 .

(2) $\operatorname{Rad} T M$ is a flat distribution on $M$.

(3) Any leaf $M^{\prime}$ of $S(T M)$ is minimal in $\bar{M}$ and has constant curvature 1.

Proof: When $M$ is totally umbilic, the last two relations in (3.1) indicate that $g(X, \zeta) H^{l}=0$ and $g(X, \zeta) H^{s}=0$, for any $X \in \Gamma(T M)$. It follows from these two relations that $H^{l}=H^{s}=0$ and hence, $M$ is totally geodesic. This was also proved in [20, Theorem 2.5, p. 6]. Therefore, in view of (2.15), we easily conclude that $\bar{R}(X, Y) Z=R(X, Y) Z$, for any $X, Y$ and $Z$ tangent to $M$. Moreover, $D^{l}=0$, so it is trivially a metric connection in this case. By the flatness assumption of $l \operatorname{tr}(T M)$ or $S\left(T M^{\perp}\right)$, we see, from Propositions 4.2 and 4.3, that $c=1$. It follows from (2) that

$$
R(X, Y) Z=\bar{R}(X, Y) Z=g(Y, Z) X-g(X, Z) Y,
$$

for all $X, Y, Z \in \Gamma(T M)$. We can easily see, from (4.8), that $M$ is a space of constant curvature 1 , which proves (1). Next, let $R^{* t}$ denote the curvature tensor of $\operatorname{Rad} T M$ with respect to $\nabla^{* t}$. Then, as per (3.8) of [9, p. 57] and the fact that $M$ is totally geodesic and of constant curvature 1 , we obtain

$$
\begin{aligned}
\bar{g}\left(R^{* t}(X, Y) \xi, N\right) & =\bar{g}(R(X, Y) \xi, N) \\
& =g(Y, \xi) \bar{g}(X, N)-g(X, \xi) \bar{g}(Y, N)=0
\end{aligned}
$$

for all $X, Y \in \Gamma(T M)$. From (4.9), we deduce that $R^{* t}=0$. Hence, $\operatorname{Rad} T M$ is flat, which proves (2). Next, assume that $S(T M)$ is integrable and let $M^{\prime}$ be its leaf. In this case, $A_{N}$ is symmetric on $S(T M)$; hence, the first relation in (1) of Lemma 3.5 helps us to get

$$
h^{*}(\bar{\phi} X, \bar{\phi} Y)=-h^{*}(X, Y) \text {, }
$$

for any $X, Y \in \Gamma(S(T M))$. Since $M$ is totally geodesic, the second fundamental form of $M^{\prime}$ in $\bar{M}$ is $h^{\prime}(X, Y)=h^{*}(X, Y)+h^{l}(X, Y)+h^{s}(X, Y)=h^{*}(X, Y)$, for any $X$ and $Y$ tangent to $M^{\prime}$. Therefore, using (4.10), we get trace ${ }_{T M^{\prime}} h^{\prime}=0$, which shows that $M^{\prime}$ is minimal. Denote by $R^{*}$ the curvature tensor of $S(T M)$ with respect to the semi-Riemannian connection $\nabla^{*}$. Then, through a direct calculation while considering (4.8), (2.10) and the fact $M$ is totally geodesic, we derive

$$
\begin{aligned}
g(Y, Z) X-g(X, Z) Y= & R(X, Y) Z=R^{*}(X, Y) Z+h^{*}\left(X, \nabla_{Y}^{*} Z\right) \\
& -h^{*}\left(Y, \nabla_{X}^{*} Z\right)-h^{*}([X, Y], Z)+\nabla_{X}^{* t} h^{*}(Y, Z)-\nabla_{Y}^{*} h^{*}(X, Z),
\end{aligned}
$$

for any $X, Y$ and $Z$ tangent to $M^{\prime}$. It follows from the relation above that

$$
R^{\prime}(X, Y) Z=g^{\prime}(Y, Z) X-g^{\prime}(X, Z) Y,
$$


AJMS

28,1

where $R^{\prime}=R_{\mid M^{\prime}}^{*}$ is the curvature tensor of $M^{\prime}$ and $g^{\prime}=g_{M^{\prime}}$. Hence, $M^{\prime}$ is a space of constant curvature 1 , which completes the proof.

Corollary 4.5. There does not exist any totally umbilic invariant lightlike submanifold of an indefinite Sasakian space form $\bar{M}(c \neq 1)$, with a flat lightlike transversal bundle or flat screen transversal bundle.

We wind up this section by giving an example of an invariant lightlike submanifold $M$ of an indefinite Sasakian manifold $\bar{M}$.

Example 4.6. (An invariant lightlike submanifold). Let $\bar{M}=\left(\mathbb{R}_{2}^{7}, \bar{\phi}, \zeta, \eta, \bar{g}\right)$ be the manifold endowed with the usual Sasakian structure (see, for example, [2, p. 321] for such a structure), in which $\bar{g}$ has signature $(-,+,+,-,+,+,+)$, with respect to the canonical basis $\left\{\partial x^{1}, \partial x^{2}, \partial x^{3}, \partial y^{1}, \partial y^{2}, \partial y^{3}, \partial z\right\}$. Suppose that $M$ is a submanifold of $\bar{M}$ given by

$$
\begin{aligned}
& x^{1}=v^{1} \cosh \theta, y^{1}=v^{2} \cos h \theta, x^{2}=v^{1} \sin h \theta-v^{2}, \\
& y^{2}=v^{1}+v^{2} \sin h \theta, x^{3}=\sin v^{3} \sin h v^{4}, y^{3}=\cos v^{3} \cos h v^{4}, z=v^{5} .
\end{aligned}
$$

It is easy to see that the vector fields $\xi_{1}, \xi_{2}, \zeta, Z_{1}, Z_{2}$, and given by

$$
\begin{aligned}
& \xi_{1}=\cos h \theta \partial x^{1}+\sin h \theta \partial x^{2}+\partial y^{2}+\left(y^{1} \cosh \theta+y^{2} \sin h \theta\right) \partial z, \\
& \xi_{2}=-\partial x^{2}+\cosh \theta \partial y^{1}+\sin h \theta \partial y^{2}-y^{2} \partial z, \quad \zeta=2 \partial z, \\
& Z_{1}=\cos v^{3} \sin h v^{4} \partial x^{3}-\sin v^{3} \cos h v^{4} \partial y^{3}+y^{3} \cos v^{3} \sin h v^{4} \partial z, \\
& Z_{2}=\sin v^{3} \cos h v^{4} \partial x^{3}+\cos v^{3} \sin h v^{4} \partial y^{3}+y^{3} \sin v^{3} \cos h v^{4} \partial z,
\end{aligned}
$$

spans $T M$. Moreover, one can see that $\operatorname{Rad} T M=\operatorname{Span}\left\{\xi_{1}, \xi_{2}\right\}$ and $S(T M)=\operatorname{Span}$ $\left\{Z_{1}, Z_{2}, \zeta\right\}$. Furthermore, we note that $\bar{\phi} \xi_{2}=\xi_{1}$ and $\bar{\phi} Z_{2}=Z_{1}$. It follows that $\operatorname{Rad} T M$ and $S(T M)$ are invariant under $\bar{\phi}$. On the other hand, $l \operatorname{tr}(T M)$ is spanned by $N_{1}$ and $N_{2}$, where

$$
\begin{aligned}
& N_{1}=2\left\{-\cos h \theta \partial x^{1}-\sin h \theta \partial x^{2}+\partial y^{2}-\left(y^{1} \cosh \theta+y^{2} \sin h \theta\right) \partial z\right\}, \\
& N_{2}=2\left\{-\partial x^{2}-\cosh \theta \partial y^{1}-\sin h \theta \partial y^{2}-y^{2} \partial z\right\} .
\end{aligned}
$$

Note that $\bar{\phi} N_{2}=N_{1}$; hence, $l \operatorname{tr}(T M)$ is invariant under $\bar{\phi}$. Therefore, $M$ is a five-dimensional invariant lightlike submanifold of $\bar{M}$.

\section{References}

[1] Duggal KL, Bejancu A. Lightlike submanifolds of semi-Riemannian manifolds and applications, Mathematics and its Applications. Dordrecht: Kluwer Academic Publishers; 1996: 364.

[2] Duggal KL, Sahin B. Differential geometry of lightlike submanifolds. Frontiers in Mathematics. Basel: Birkhauser; 2010.

[3] Atindogbé C. Scalar curvature on lightlike hypersurfaces. Balkan Society of Geometers. Appl Sci. 2009; 11: 9-18.

[4] Atindogbe C, Duggal KL. Conformal screen on lightlike hypersurfaces. Int J Pure and Applied Math. 2004; 11(4): 421-442.

[5] Atindogbe C, Ezin JP, Tossa J. Reduction of the codimension for lightlike isotropic submanifolds. J Geom Phys. 2002; 42: 1-11. doi: 10.1016/S0393-0440(01)00027-4. 
[6] Bejancu A, Deshmukh S. The transversal vector bundle of a lightlike Finsler submanifold. Arab J Math Sci. 1997; 3(2): 37-51.

[7] Bejancu A, Duggal KL. Lightlike submanifolds of semi-Riemannian. Acta Appl Math 1995; 38(2): 197-215. doi: 10.1007/BF00992847.

[8] Calin C, Contributions to geometry of CR-submanifold, Thesis. Romania: University of Iasi; 1998.

[9] Duggal KL, Jin DH. Totally umbilical lightlike submanifolds. Kodai Math J. 2003; 26: 49-68. Available from: https://projecteuclid.org/euclid.kmj/1050496648.

[10] Gupta RS, Sharfuddin A. Generalised Cauchy-Riemann lightlike submanifolds of indefinite Kenmotsu manifolds. Note Mat. 2010; 30(2): 49-59. doi: 10.1285/115900932v30n2p49.

[11] Izumiya S. Lightlike hypersurfaces along spacelike submanifolds in anti-de Sitter space. J Math Phys. 2015; 56(11): 112502. 29. doi: 10.1063/1.4936148.

[12] Sahin B, Yildirim C. Slant lightlike submanifolds of indefinite Sasakian manifolds. Filomat. 2012; 26(2): 277-287. doi: 10.2298/FIL1202277S.

[13] Ssekajja S. A note on null hypersurfaces of indefinite Kaehler space forms. Balkan J Geometry Appl. 2020; 25(2): 94-105.

[14] Ssekajja S. Geometry of isoparametric null hypersurfaces of lorentzian manifolds. J Korean Math Soc. 2020; 571: 195-213. doi: 10.4134/JKMS.j190001.

[15] Ssekajja S. Some results on null hypersurfaces in (LCS)-manifolds. Kyungpook Math. J. 2019; 59: 783-795. doi: 10.5666/KMJ.2019.59.4.783.

[16] Wang Z, Pei D, Chen L. Geometry of 1-lightlike submanifolds in anti-de Sitter n-space. Proc Roy Soc. Edinburgh Sect. A. 2013; 143(5): 10891113.

[17] De UC, Majhi P. On invariant submanifolds of Kenmotsu manifolds. J Geom. 2015; 106: 109-122. doi: 10.1007/s00022-014-0238-y.

[18] Karadag HB, Atceken M. Invariant submanifolds of Sasakian manifolds. Balkan J Geometry Appl. 2007; 12(1): 68-75.

[19] Kon M. Invariant submanifolds of normal contact metric manifolds. Kodai Math Sem Rep. 1973; 25(3): 330-336. Available from: https://projecteuclid.org/euclid.kmj/1138846821.

[20] Duggal KL, B. Sahin. Lightlike submanifolds of indefinite Sasakian manifolds. Int J Math Math Sci. 2007: 21. 57585. doi: 10.1155/2007/57585.

[21] Blair DE. Riemannian geometry of contact and symplectic manifolds. 2nd ed. Boston: Birkhäuser; 2010.

[22] Kupeli DN. Singular semi-riemannian geometry. In: Mathematics and Applications. Dordrecht: Kluwer Academic Publishers. 1996; 366.

\section{Corresponding author}

Samuel Ssekajja can be contacted at: samuel.ssekajja@wits.ac.za
Invariant lightlike submanifolds 